\title{
SOME SPECTRAL PROPERTIES OF AN INTEGRAL OPERATOR IN POTENTIAL THEORY
}

\author{
by JOHN F. AHNER
}

(Received 4th September 1985)

\section{Introduction}

In [7] Plemelj established some fundamental results in two- and three-dimensional potential theory about the eigenvalues of both the double layer potential operator and its adjoint, the normal derivative of the single layer potential operator. In [3] Blumenfeld and Mayer established some additional results concerning the eigenvalues of these integral operators in the case of $\mathbb{R}^{2}$. The spectral properties established by Plemelj [7] and by Blumenfeld and Mayer [3] have had a profound effect in the area of integral equation methods in scattering and potential theory in both $\mathbb{R}^{2}$ and $\mathbb{R}^{3}$.

Some applications that have been made of these results may be found in Colton and Kress [4]. A complete list, however, of all the different uses that have been made of the efforts of Plemelj [7] and of Blumenfeld and Mayer [3] would be a formidable task.

This paper arose from the author's long interest in the spectral properties of the double layer potential integral operator in both $\mathbb{R}^{2}$ and $\mathbb{R}^{3}$. For sufficiently smooth boundaries, it can be shown that for both $\mathbb{R}^{2}$ and $\mathbb{R}^{3}$ the point 0 lies in the spectrum of the integral operator. A fundamental question is what part of the spectrum does 0 lie in?

For the case of $\mathbb{R}^{3}$ some partial results on this topic are known. If the underlying boundary is either a sphere or a prolate spheroid, it can be shown (see [1]) that the eigenvalues of the double layer potential integral operator lie in the interval $[-1,0)$. Furthermore, it can be established that for both geometries 0 lies in the continuous spectrum of the integral operator. As for other geometries, the spectral classification of 0 remains an open question.

For the case of $\mathbb{R}^{2}$, it turns out that the situation is somewhat different for the double layer potential operator, which in this paper we denote by $K$ and define in equation (2.1). After encountering some serious difficulties in an attempt to establish a general theorem about which part of the spectrum of $K$ the point 0 lies in, the author looked at some specific examples, namely, the circle and the ellipse. For the case of the circle, the point 0 lies in the point spectrum of $K$. For the case of an ellipse, 0 lies in the continuous spectrum of $K$. Consequently, these examples demonstrate that 0 does not always lie in the same component of the spectrum of $K$ for all sufficiently smooth boundaries.

In the next section we give some notation, definitions and basic results which we shall use. In Section 3, we consider the case of a circle and compute the spectrum of $K$. It is shown that 0 is an eigenvalue and moreover, that it has infinite geometric multiplicity. 
In Section 4 we consider the case of an ellipse. There we compute all the eigenvalues for $K$ and establish that every eigenvalue has a geometric multiplicity of 1 . Furthermore, we prove that the continuous spectrum of $K$ is equal to the set $\{0\}$.

\section{Notation, definitions and basic results}

In this section we give our notation and state some definitions and results which we shall require. Let $D_{i}$ be a bounded, simply connected domain in $\mathbb{R}^{2}$ containing the origin with a $C^{2}$ boundary $\partial D$, and let $D_{e}$ denote the region exterior to $\bar{D}_{i}$. Let $\hat{n}$ denote a unit normal directed out of $D_{i}$. Let $x$ and $y$ denote typical points in $\mathbb{R}^{2}$.

We now define the following integral operators of potential theory:

$$
\begin{gathered}
(K \psi)(x):=\frac{1}{\pi} \int_{\partial D} \psi(y) \frac{\partial}{\partial n(y)} \ln \frac{1}{|x-y|} d s_{y}, \quad x \in \partial D, \\
(D \psi)(x):=\frac{1}{\pi} \int_{\partial D} \psi(y) \frac{\partial}{\partial n(y)} \ln \frac{1}{|x-y|} d s_{y}, \quad x \in \mathbb{R}^{2} \backslash \partial D .
\end{gathered}
$$

Here it is understood that the integration is taken with respect to arc length.

A standard result in two-dimensional potential theory (e.g. see [9, pp. 78-80]) states that for closed smooth curves $\partial D$

$$
\lim _{\substack{x \rightarrow y \\ x, y \in D D}} \frac{\partial}{\partial n(y)} \ln \frac{1}{|x-y|}=-\frac{1}{2} \kappa(y),
$$

where $\kappa(y)$ denotes the curvature of $\partial D$ at $y$. Consequently, unlike the weakly singular nature of the double layer kernel in $\mathbb{R}^{3}$, the double layer kernel in $\mathbb{R}^{2}$ is continuous for all points $x$ and $y$ on $\partial D$, including when $x=y$.

Let $C(\partial D)$ denote the Banach space of complex-valued, continuous functions defined on $\partial D$ equipped with the maximum norm. Since the integral operator $K$ has a continuous kernel, it follows that $K$ is a compact linear operator on $C(\partial D)$ (see [4, Theorem 1.10]).

Let + and - denote the limits obtained for the double layer potential $(D \psi)(x)$ by approaching the boundary $\partial D$ from $D_{e}$ and $D_{i}$, respectively, that is

$$
\begin{aligned}
& \left(D_{+} u\right)(x)=\lim _{\substack{x \rightarrow x \\
x_{e} \in D_{e}}}(D u)\left(x_{e}\right), \quad x \in \partial D, \\
& \left(D_{-} u\right)(x)=\lim _{\substack{x_{i} \rightarrow x \\
x_{i} \in D_{i}}}(D u)\left(x_{i}\right), \quad x \in \partial D .
\end{aligned}
$$

It can be shown (e.g. see $[5$, p. 49] or $[8$, p. 392]) that

$$
\left(D_{ \pm} u\right)(x)=(K u)(x) \pm u(x), \quad x \in \partial D .
$$


Let $A$ denote any bounded linear operator mapping a Banach space $X$ into itself. By an eigenvalue of $A$ we mean a complex number $\lambda$ such that the nullspace $N(\lambda I-$ $A) \neq\{0\}$ where $I$ denotes the identity operator. Let $\rho(A)$ denote the resolvent set of $A$. Let $\sigma(A)$ denote the spectrum of $A$. Let $\sigma_{C}(A), \sigma_{P}(A)$, and $\sigma_{R}(A)$ denote the continuous spectrum, point spectrum, and residual spectrum of $A$, respectively. It is known (e.g. see [2, Chapter 18] or [4, Theorem 1.34]) that if $X$ is an infinite dimensional Banach space and if $A$ is a compact linear operator then $\lambda=0$ lies in $\sigma(A)$ and $\sigma(A) \backslash\{0\}$ consists of at most a countable number of eigenvalues, with $\lambda=0$ the only possible limit point.

It can be shown (see [3]) that the eigenvalues of the integral operator $K$, defined in equation (2.1), lie in the interval $[-1,1)$ and are symmetric with respect to the origin. The only exception is the eigenvalue -1 corresponding to constant eigenfunctions.

Finally, we shall denote the set of positive integers by $\mathbb{N}$.

\section{The Circle}

In this section $\partial D$ is taken to be a circle of radius $a$. Under this assumption, we compute the spectrum of the integral operator $K$ and also determine the spectral properties of the point $\lambda=0$ for $K$.

With respect to polar coordinates, let the points $x$ and $y$ be given by $\left(r_{x}, \phi_{x}\right)$ and $(r, \phi)$, respectively. Then

$$
\ln \frac{1}{|x-y|}=-\frac{1}{2} \ln \left[r_{x}^{2}+r^{2}-2 r_{x} r \cos \left(\phi-\phi_{x}\right)\right]
$$

From equation (3.1), for $x, y \in \partial D$, we have the following known result (e.g. see [5, p. 52])

$$
\begin{aligned}
\frac{\partial}{\partial n(y)} \ln \frac{1}{|x-y|} & =\frac{\partial}{\partial r}\left\{-\frac{1}{2} \ln \left[a^{2}+r^{2}-2 a r \cos \left(\phi-\phi_{x}\right)\right]\right\}_{r=a} \\
& =-\frac{1}{2 a}
\end{aligned}
$$

Before proceeding to the stated purposes of this section, it is worthy of note to examine the result in equation (3.2) in the context of equation (2.3). It is a well known result in differential geometry that the curvature of a circle of radius $a$ is $1 / a$. Consequently, the results in equations (2.3) and (3.2) are seen to be compatible.

From equations (2.1) and (3.2) it follows that

$$
K \psi(x)=-\frac{1}{2 \pi} \int_{0}^{2 \pi} \psi(a, \phi) d \phi .
$$

Letting $\psi$ equal $1, \cos m \phi$, and $\sin m \phi, m \in \mathbb{N}$, respectively, in equation (3.3) we have

$$
K(1)=-1
$$




$$
K(\cos m \phi)=K(\sin m \phi)=0 .
$$

Thus $\lambda=-1$ is an eigenvalue of $K$ with corresponding eigenfunction 1 , and $\lambda=0$ is also an eigenvalue of $K$ with corresponding eigenfunctions $\{\cos m \phi, \sin m \phi: m \in \mathbb{N}\}$. From the completeness of the orthogonal set of eigenfunctions $\{1, \cos m \phi, \sin m \phi: m \in \mathbb{N}\}$ in $L^{2}[0,2 \pi]$, it follows, by a similar argument as used in [1], that with respect to the underlying Banach space $C(\partial D)$

$$
\sigma_{P}(K)=\{-1,0\}
$$

That is, $\lambda=-1$ and $\lambda=0$ are the only eigenvalues of $K$ for the case when $\partial D$ is a circle.

In view of the fact that $K$ is a compact linear operator on $C(\partial D)$, it follows that if $\lambda \neq 0$ then either $\lambda \in \rho(K)$ or $\lambda \in \sigma_{P}(K)$. Consequently, $\lambda=-1$ and $\lambda=0$ are the only elements of $\sigma(K)$. Furthermore, since $\cos m \phi$ and $\sin m \phi, m \in \mathbb{N}$, are all eigenfunctions of $K$ corresponding to $\lambda=0$, it follows that

$$
\operatorname{dim} N(K)=\infty .
$$

\section{The ellipse}

The elliptical coordinates $(\mu, \phi)$ are related to the rectangular Cartesian coordinates $\left(y_{1}, y_{2}\right)$ by the transformation

$$
\begin{gathered}
y_{1}=\frac{1}{2} c \cosh \mu \cos \phi, \\
y_{2}=\frac{1}{2} c \sinh \mu \sin \phi,
\end{gathered}
$$

where $0 \leqq \mu<\infty, 0 \leqq \phi \leqq 2 \pi$. The closed curves corresponding to $\mu=$ constant, $0 \leqq \phi \leqq 2 \pi$ are confocal ellipses of interfocal distance $c$, eccentricity $e=(\cosh \mu)^{-1}$, major axis $c \cosh \mu$ and minor axis $c \sinh \mu$. The limiting case $\mu=0$ represents the line segment between the foci.

In this section $\partial D$ will denote the ellipse corresponding to $\mu=b, 0 \leqq \phi \leqq 2 \pi$, where $b$ is some constant. To avoid the degenerate case, we will assume that $b>0$.

In terms of elliptical coordinates it can be shown that the gradient of a scalar function $\Phi(\mu, \phi)$ is given by

$$
\nabla \Phi(\mu, \phi)=\frac{2}{c \tau}\left(\frac{\partial \Phi}{\partial \mu} \hat{e}_{\mu}+\frac{\partial \Phi}{\partial \phi} \hat{e}_{\phi}\right)
$$

where

$$
\tau:=\left[\cosh ^{2} \mu \sin ^{2} \phi+\sinh ^{2} \mu \cos ^{2} \phi\right]^{1 / 2},
$$


and where $\hat{e}_{\mu}$ and $\hat{e}_{\phi}$ denote the orthonormal vectors

$$
\begin{aligned}
& \hat{e}_{\mu}:=(\sinh \mu \cos \phi \hat{i}+\cosh \mu \sin \phi \hat{j}) / \tau \\
& \hat{e}_{\phi}:=(-\cosh \mu \sin \phi \hat{i}+\sinh \mu \cos \phi \hat{j}) / \tau
\end{aligned}
$$

Furthermore, it can be shown that the element of arc length $d s$ is given by

$$
d s=\frac{c}{2} \tau d \phi
$$

From [6, p. 1202] we have

$$
\begin{aligned}
\ln \frac{1}{|x-y|}= & -\left(\mu_{>}+\ln \frac{c}{4}\right)+\sum_{n=1}^{\infty} \frac{2}{n} e^{-n \mu_{>}}\left[\cosh n \mu_{<} \cos n \phi \cos n \phi_{x}\right. \\
& \left.+\sinh n \mu_{<} \sin n \phi \sin n \phi_{x}\right],
\end{aligned}
$$

where $\mu_{>}=\max \left\{\mu_{x}, \mu_{y}\right\}, \mu_{<}=\min \left\{\mu_{x}, \mu_{y}\right\}$, and $\left(\mu_{x}, \phi_{x}\right)$ and $\left(\mu_{y}, \phi\right)$ denote the elliptical coordinates of the points $x$ and $y$, respectively.

At the point $(b, \phi) \in \partial D$ the unit tangent vector $\hat{T}$ and the outer unit normal vector $\hat{n}$ are given, respectively, by

$$
\hat{T}=\hat{e}_{\phi}, \quad \hat{n}=\hat{e}_{\mu} .
$$

For $y=(b, \phi) \in \partial D$ and $x=\left(\mu_{x} \phi_{x}\right) \in D_{i}$ it follows from equations (4.2) and (4.6) that

$$
\begin{gathered}
\frac{\partial}{\partial n(y)} \ln \frac{1}{|x-y|}=\frac{-2}{c \tau}\left\{1+2 \sum_{n=1}^{\infty} e^{-n b}\left[\cosh n \mu_{x} \cos n \phi \cos n \phi_{x}\right.\right. \\
\left.\left.+\sinh n \mu_{x} \sin n \phi \sin n \phi_{x}\right]\right\}
\end{gathered}
$$

Consequently, from equations (2.4), (4.5) and (4.8) we have

$$
\begin{gathered}
D \psi(x)=-\frac{1}{\pi} \int_{0}^{2 \pi} \psi(\mu, \phi)\left\{1+2 \sum_{n=1}^{\infty} e^{-n b}\left[\cosh n \mu_{x} \cos n \phi \cos n \phi_{x}\right.\right. \\
\left.\left.+\sinh n \mu_{x} \sin n \phi \sin n \phi_{x}\right]\right\} d \phi, \quad x \in D_{i} .
\end{gathered}
$$

Letting $\psi$ equal $1, \cos m \phi$, and $\sin m \phi$, where $m \in \mathbb{N}$, respectively, in equation (4.9), then using the orthogonality of the trigonometric functions, and finally letting 
$\mu_{x} \rightarrow b$, we have from equation (2.6)

$$
\begin{gathered}
K(1)=-1, \\
K(\cos m \phi)=-e^{-2 m b} \cos m \phi, \quad m \in \mathbb{N}, \\
K(\sin m \phi)=e^{-2 m b} \sin m \phi, \quad m \in \mathbb{N}
\end{gathered}
$$

Thus it is seen that $-1,-\mathrm{e}^{-2 m b}$, and $e^{-2 m b}, m \in \mathbb{N}$, are eigenvalues of $K$ with corresponding eigenfunctions $1, \cos m \phi$, and $\sin m \phi$, respectively. From the completeness of the orthogonal set of eigenfunctions $\{1, \cos m \phi, \sin m \phi: m \in \mathbb{N}\}$ in $L^{2}[0,2 \pi]$, it follows, by an argument similar to one used in [1], that with respect to the underlying Banach space $C(\partial D)$

$$
\sigma_{P}(K)=\left\{-1, \pm e^{-2 m b}: m \in \mathbb{N}\right\}
$$

That is, the above eigenvalues are the only eigenvalues of $K$. Consequently, unlike the situation when $\partial D$ is a circle,

$$
0 \notin \sigma_{P}(K)
$$

when $\partial D$ is an ellipse. Furthermore, it is seen that

$$
\operatorname{dim} N(-I-K)=\operatorname{dim} N\left( \pm e^{-2 m b} I-K\right)=1
$$

for each $m \in \mathbb{N}$. Therefore each eigenvalue has a geometric multiplicity of 1 .

To complete the analysis of this section we establish the following result which determines the spectral nature of the point $\lambda=0$.

Theorem 4.1 Let $\partial D$ denote the ellipse corresponding to $\mu=b, 0 \leqq \phi \leqq 2 \pi$, where $b$ is some positive constant. Then

$$
\{0\}=\sigma_{C}(K)
$$

Proof. Since $K$ is a compact linear operator on $C(\partial D)$,

$$
0 \in \sigma(K)
$$

Furthermore, since each eigenfunction must necessarily lie in the range of $K, R(K)$, we have

$$
\{1, \cos m \phi, \sin m \phi: m \in \mathbb{N}\} \subset R(K) .
$$

It follows that $R(K)$ is dense in $C(\partial D)$. Consequently, from equations (4.14) and (4.16) it 
follows that

$$
0 \in \sigma_{C}(K)
$$

Finally, by using the fact that $K$ is a compact linear operator on $C(\partial D)$, we have from equation (4.18)

$$
\{0\}=\sigma_{c}(K) \text {. }
$$

\section{REFERENCES}

1. J. F. Ahner and R. F. Arenstorf, On the eigenvalues of the electrostatic integral operator, $J$. Math. Anal. Appl., in press.

2. G. Bachman and L. Narici, Functional Analysis (Academic Press, New York, 1966).

3. J. Blumenfeld and W. MAYer, Über poincarésche fundamentalfunktionen, Sitz. Wien. Akad. Wiss., Math.-Nat. Klasse 122, Abt. IIa (1914), 2011-2047.

4. D. Colton and R. KRess, Intergral Equation Methods in Scattering Theory (Wiley, New York, 1983).

5. M. A. JAswon and G. T. SYMm, Integral Equation Methods in Potential Theory and Elastostatics (Academic Press, New York, 1977).

6. P. M. Morse and H. Feshbach, Methods of Theoretical Physics (McGraw-Hill, New York, 1953).

7. J. Plemel, Potentialtheoretische Untersuchungen (Preisschriften der Fürstlich Jablonowskischen Gesellschaft zu Leipzig, Teubner-Verlag, Leipzig, 1911).

8. A. N. Tikhonov and A. A. SamarskiI, Equations of Mathematical Physics (Pergamon Press, New York, 1963).

9. F. G. Tricomi, Integral Equations (Wiley, New York, 1957).

Department of Mathematics

VANDERBILT UNIVERSITY

Nashyille, Tennessee 37235 